\title{
Thienemannimyia (Thienemannimyia) sinogalbina nom. n., a replacement name for Thienemannimyia galbina Cheng \& Wang, 2009
}

\author{
Xiao-Long Lin* and Xin-Hua Wang \\ College of Life Sciences, Nankai University, Tianjin, 300071, China. \\ "Corresponding author. E-mail: lin880224@gmail.com \\ http://zoobank.org/FBE357A4-BF71-499B-98F6-A3CE25D228C0
}

\begin{abstract}
The recent transfer of Hayesomyia galbina Cheng \& Wang, 2006 to Thienemannimyia (Hayesomyia) created a homonymy with Thienemannimyia (Thienemannimyia) galbina Cheng \& Wang, 2009. Thienemannimyia (Hayesomyia) galbina (Cheng \& Wang, 2006) has priority and Thienemannimyia (Thienemannimyia) sinogalbina nom. n. is proposed as a new replacement for the junior secondary homonymy Thienemannimyia (Thienemannimyia) galbina Cheng \& Wang, 2009.
\end{abstract}

\section{Introduction}

Cheng and Wang (2006) described Hayesomyia galbina, and Cheng and Wang (2009) described Thienemannimyia galbina based on adult males from China. According to recent morphological phylogeny of Tanypodinae, Silva and Ekrem (2016) treated Hayesomyia as a subgenus of Thienemannimyia. Hence Thienemannimyia (Thienemannimyia) galbina Cheng \& Wang, 2009 was rendered a junior secondary homonymy.

According to Article 53.3 of the International Code of Zoological Nomenclature (ICZN 1999), Thienemannimyia (Hayesomyia) galbina (Cheng \& Wang, 2006) has priority and a new name Thienemannimyia (Thienemannimyia) sinogalbina nom. n. is proposed for Thienemannimyia (Thienemannimyia) galbina Cheng \& Wang, 2009.

\section{Abbreviations}

NKU: College of Life Sciences, Nankai University.

\section{Thienemannimyia (Thienemannimyia) sinogalbina nom. n.}

Thienemannimyia galbina Cheng \& Wang, 2009: 54, figs 14-16.

http://zoobank.org/FBE357A4-BF71-499B-98F6-A3CE25D228C0

Holotype: (NKU No. 02260) China, Fujian, Wuyishan, Wuyi Mountain Natural Conversation, Sangan, 24.iv.1993, light trap, X.H. Wang. Paratype: 1 male (NKU N0. 11728) China, Sichuan, Shimian County, near Nanya River, 16.vi.1996, light trap, X.H. Wang.

Distribution. China.

Etymology. The new name consists of the original species name with the prefix 'sino' indicating the currently known distribution, China.

\section{Acknowledgements}

Financial support from the National Natural Science Foundation of China (NSFC, No. 31272284) is gratefully acknowledged.

\section{References}

ICZN (1999) International Code of Zoological Nomenclature. Fourth edition. International Trust for Zoological Nomenclature, London, $306 \mathrm{pp}$.

Cheng, M. and Wang, X.H. (2006) Review of Hayesomyia Murray \& Fittkau (Diptera: Chironomidae: Tanypodinae) from China. - Zootaxa 1147: 35-59. DOI: http://dx.doi.org/10.11646/zootaxa.1147.1.2 
Cheng, M. and Wang, X. (2009) Thienemannimyia Fittkau (Diptera: Chironomidae) from China. - Zootaxa 2074: 50-60. DOI: http://dx.doi.org/10.11646/zootaxa.2074.1.2

Silva, F.L. and Ekrem, T. (2016) Phylogenetic relationships of nonbiting midges in the subfamily Tanypodinae (Diptera: Chironomidae) inferred from morphology. - Systematic Entomology 41, 73-92. DOI: https://dx.doi.org/10.1111/syen.12141

Article submitted 8. October 2019, accepted by Torbjørn Ekrem 13. October 2019, published 16. October 2019. 